Genetics and population analysis

\title{
A system for exact and approximate genetic linkage analysis of SNP data in large pedigrees
}

Mark Silberstein, Omer Weissbrod, Lars Otten, Anna Tzemach, Andrei Anisenia, Oren Shtark, Dvir Tuberg, Eddie Galfrin, Irena Gannon, Adel Shalata, Zvi U. Borochowitz, Rina Dechter, Elizabeth Thompson and Dan Geiger

Vol. 29, No. 2, 2013, pp. 197-205

doi:10.1093/bioinformatics/bts658

The publishers regret that the author affiliations for this paper should appear as follows:

Mark Silberstein ${ }^{1,2}$, Omer Weissbrod ${ }^{1, *}$, Lars Otten ${ }^{3}$, Anna Tzemach $^{1}$, Andrei Anisenia ${ }^{1,4}$, Oren Shtark ${ }^{1}$, Dvir Tuberg ${ }^{1}$, Eddie Galfrin ${ }^{1}$, Irena Gannon ${ }^{1}$, Adel Shalata ${ }^{5,6,7}$, Zvi U. Borochowitz $^{5,8}$, Rina Dechter ${ }^{3}$, Elizabeth Thompson ${ }^{9}$ and Dan Geiger $^{1}$

${ }^{1}$ Department of Computer Science, Technion-Israel Institute of Technology, Haifa, Israel, ${ }^{2}$ Department of Computer Science,
University of Texas at Austin, Austin, TX, USA, ${ }^{3}$ Donald Bren School of Information and Computer Sciences, UC Irvine, CA, USA, ${ }^{4}$ Department of Computer Science, University of Ottawa, Ottawa, Canada, ${ }^{5}$ The Simon Winter Institute for Human Genetics, Bnai-Zion Medical Center, Haifa, Israel, ${ }^{6}$ Research and Development Center, The Galilee Society, Shefa-Amr, Israel, ${ }^{7}$ Holy Family Hospital, Nazareth, Israel, ${ }^{8}$ The Rappaport Faculty of Medicine and Research Institute, Technion-Israel Institute of Technology, Haifa, Israel and ${ }^{9}$ Department of Statistics, University of Washington, Seattle, WA, USA 